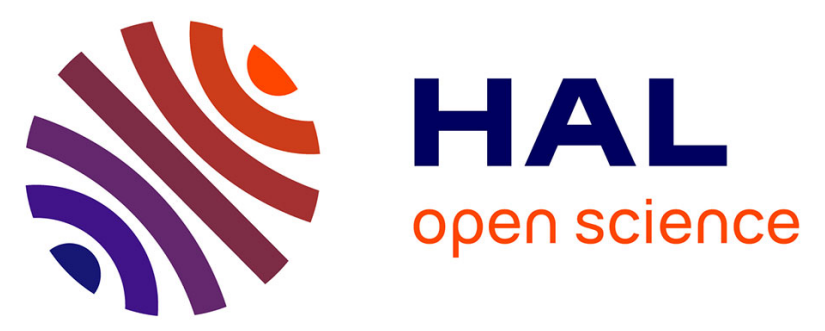

\title{
iCHORD-SI combination as an alternative to EDS-EBSD coupling for the characterization of $\gamma-\gamma^{\prime}$ nickel-based superalloy microstructures
}

Suzanne Vernier, Jean-Michel Franchet, Maxime Lesne, Thierry Douillard, Jeremie Silvent, Cyril Langlois, Nathalie Bozzolo

\section{To cite this version:}

Suzanne Vernier, Jean-Michel Franchet, Maxime Lesne, Thierry Douillard, Jeremie Silvent, et al.. iCHORD-SI combination as an alternative to EDS-EBSD coupling for the characterization of $\gamma$ $\gamma^{\prime}$ nickel-based superalloy microstructures. Materials Characterization, 2018, 142, pp.492 - 503. 10.1016/j.matchar.2018.06.015 . hal-01822412

HAL Id: hal-01822412

https:/ /hal-mines-paristech.archives-ouvertes.fr/hal-01822412

Submitted on 18 Jul 2018

HAL is a multi-disciplinary open access archive for the deposit and dissemination of scientific research documents, whether they are published or not. The documents may come from teaching and research institutions in France or abroad, or from public or private research centers.
L'archive ouverte pluridisciplinaire HAL, est destinée au dépôt et à la diffusion de documents scientifiques de niveau recherche, publiés ou non, émanant des établissements d'enseignement et de recherche français ou étrangers, des laboratoires publics ou privés. 


\title{
iCHORD-SI combination as an alternative to EDS-EBSD coupling for the characterization of $\gamma$ - $\gamma^{\prime}$ nickel-based superalloy microstructures
}

\author{
Suzanne Vernier ${ }^{\mathrm{a}, \mathrm{b}^{*}}$, Jean-Michel Franchet ${ }^{\mathrm{b}}$, Maxime Lesne ${ }^{\mathrm{c}, \mathrm{d}}$, Thierry Douillard ${ }^{\mathrm{d}}$, Jérémie \\ Silvent $^{\mathrm{c}}$, Cyril Langlois ${ }^{\mathrm{d}}$ and Nathalie Bozzolo ${ }^{\mathrm{a}}$
}

\author{
${ }^{a}$ MINES ParisTech, PSL - Research University, CEMEF - Centre de mise en forme des matériaux, CNRS UMR 7635, 1 rue Claude \\ Daunesse, 06904 Sophia Antipolis, France \\ ${ }^{\mathrm{b}}$ Safran SA, SafranTech - Materials \& Process Department, 1 Rue Geneviève Aube, 78114 Magny-les-Hameaux, France \\ ${ }^{c}$ Orsay Physics - Tescan Orsay Holding, 95 Avenue des Monts Auréliens, ZA Saint-Charles - F, 13710 Fuveau, France \\ ${ }^{\mathrm{d}}$ University of Lyon, INSA de Lyon, MATEIS CNRS UMR5510, Bât. Blaise Pascal, 20 Avenue Albert Einstein, 69621 Villeurbanne, France \\ *Corresponding author. Suzanne.vernier@mines-paristech.fr
}

\begin{abstract}
Because they exhibit very similar diffraction patterns, the $\gamma$ and $\gamma^{\prime}$ phases of nickel-based superalloys cannot be distinguished by the conventional EBSD technique alone. This paper presents an original method which discriminates those phases on an orientation map by exploiting their chemical differences. Performed with a Focused Ion Beam (FIB) microscope, the method is the combination of the ion CHanneling ORientation Determination technique (iCHORD) - recently developed by Langlois et al. [1] - with the chemical information obtained from Secondary Ion (SI) images. The performances of the method are compared to the EDS-EBSD coupling which also discriminates phases based on their chemical compositions. Applied filters, angular resolution, spatial resolution of the phase discrimination and acquisition time are discussed. It results that the iCHORD-SI combination offers an orientation map with an angular resolution slightly decreased compared to that of the EBSD technique, but with an interesting phase resolution (down to $150 \mathrm{~nm}$ ) and within a reasonable acquisition time. Thus, the iCHORD-SI combination appears to be an interesting method for the crystallographic systems where phases are difficult to discriminate by the EBSD technique but present a significant chemical contrast.
\end{abstract}

Keywords. Focused Ion Beam; Ion channeling; Secondary ions; EDS-EBSD coupling; Gamma - Gamma Prime nickel-based superalloy.

\section{Introduction}

Due to their superior resistance at high temperature, $\gamma-\gamma^{\prime}$ nickel-based superalloys are used for the manufacturing of highly-constrained aero-engine parts [2]. Their mechanical properties at high temperature result from several features including the $\gamma^{\prime}$ phase distribution. Indeed, the $\gamma^{\prime}$ phase forms precipitates of different sizes which are spread inside the $\gamma$ matrix, the finer precipitates ensuring the strengthening of the alloy [3]. But many interactions between the $\gamma$ grains and the $\gamma^{\prime}$ precipitates also occur during hot forging operations: deformation triggers recrystallization mechanisms which closely depend on the precipitation and its evolution. For instance, precipitates may dissolve to potentially reprecipitate behind the front [4,5], pin grain boundaries [6] or act as nucleation sites [7,8]. Understanding these $\gamma$ $\gamma^{\prime}$ interactions enables the optimization of the forging process so as to obtain the $\gamma^{\prime}$ phase distribution leading to the best mechanical properties. Thus, for the $\gamma-\gamma^{\prime}$ microstructures the characterization of respective phases is a real need. 
Electron Back-Scattered Diffraction (EBSD) is commonly used to characterize polycrystalline microstructures. However, in the case of the $\gamma-\gamma^{\prime}$ microstructures, this technique is not able to discriminate the $\gamma$ and $\gamma^{\prime}$ phases because of their respective crystallographic structures, FCC and $\mathrm{L}_{2}$, which lead to very similar diffraction patterns [9]. Superstructure reflections, which are theoretically present in the $\gamma^{\prime}$ patterns and absent in those of the $\gamma$ phase, have too low intensities to be detected with the conventional EBSD mapping settings (e.g. diffraction patterns of 160x120 pixels).

$\gamma^{\prime}$ phase is easily recognizable on BackScattered Electron (BSE) images because its higher content in light chemical elements makes it appear darker than the $\gamma$ phase. The overlay of a BSE image acquired at a $0^{\circ}$ tilt angle with an EBSD map acquired at a $70^{\circ}$ tilt is difficult since distortions are very likely to exist between the two images even after tilt correction. In the EBSD sample configuration, BSE can be collected either by a BSE detector beneath the SEM column or by a solid-state BSE detector mounted at the topside of the EBSD camera. But in both cases the collected intensity is low, which can result in noisy images [10]. In addition, with the solid-state detector optimal contrast settings can be difficult to find [10]. In order to collect more intensity, some research have been done in order to use the EBSD detector itself as a BSE detector [10]; but the technique is not widespread.

Performing Energy Dispersive X-ray Spectrometry (EDS) at the same time as the EBSD scan allows the discrimination of the phases thanks to their difference in chemical composition [11,12]. One limit of this method is the spatial resolution of the EDS technique which depends on the chemical composition of the material as well as on the electron beam energy $[13,14]$. Yet, $\gamma^{\prime}$ precipitates as small as $100 \mathrm{~nm}$ must be considered in the analysis of the microstructures because they have a significant effect on the mechanisms and kinetics of recrystallization in addition to their impact on the material mechanical properties.

The present paper aims at presenting another method to discriminate the $\gamma$ and $\gamma^{\prime}$ phases on an orientation map, also based on the chemical differences of the phases but performed with a Focused Ion Beam (FIB) microscope. This methods combines the ion CHanneling ORientation Determination (iCHORD) technique, recently developed by Langlois et al. [1] and which uses the intensity variations in Secondary Electron (SE) images to determine the crystal orientations, with the Secondary Ion (SI) signal whose intensity strongly depends on the atomic number [15]. Acquired with the same scan electronics and without changing the sample position, SE and SI signals are can be easily superimposed, providing an orientation map with phase identification. The performances of the iCHORD-SI combination on a $\gamma-\gamma$ ' microstructure are analyzed in regards to that of the EDS-EBSD coupling. Applied filters (part 3.1), angular resolution (part 3.2), spatial resolution of the phase discrimination (part 3.3) and acquisition time (part 3.4) are discussed.

\section{Experimental}

\subsection{Material}

The AD $730^{\mathrm{TM}}$ alloy is a polycrystalline $\gamma-\gamma$ ' nickel-based superalloy recently designed by the Aubert\&Duval Company for aero-engine applications [16]. Its chemical composition is given in Table 1.

Table 1. Composition of the AD730 ${ }^{T M}$ nickel-based superalloy (wt\%) [16].

\begin{tabular}{|c|c|c|c|c|c|c|c|c|c|c|c|c|}
\hline Element & $\mathbf{N i}$ & $\mathrm{Fe}$ & Co & $\mathrm{Cr}$ & Mo & W & Al & $\mathbf{T i}$ & $\mathbf{N b}$ & B & C & $\mathbf{Z r}$ \\
\hline AD730 ${ }^{\mathrm{TM}}$ & Base & 4.00 & 8.50 & 15.70 & 3.10 & 2.70 & 2.25 & 3.40 & 1.10 & 0.01 & 0.015 & 0.03 \\
\hline
\end{tabular}

The $\gamma$ phase forms the matrix of the alloy and is a Face Centered Cubic solid solution. The $\gamma^{\prime}$ phase forms precipitates of various sizes and has a Simple Cubic $\mathrm{L}_{2}$ ordered structure. The 
chemical composition of each phase measured on the as-received billet by EDS at $15 \mathrm{kV}$ is presented in Table 2 .

Table 2. Compositions of the $\gamma$ and $\gamma^{\prime}$ phases measured on the as-received billet by EDS at $15 \mathrm{kV}$ (wt\% - semiquantitative values obtained without using a suitable standard-average values over an area of few $\mu^{2}{ }^{2}$ ).

\begin{tabular}{|c|c|c|c|c|c|c|c|c|c|c|c|c|}
\hline Element & $\mathbf{N i}$ & $\mathbf{F e}$ & Co & $\mathrm{Cr}$ & Mo & $\mathbf{W}$ & Al & $\mathbf{T i}$ & $\mathbf{N b}$ & B & $\mathbf{C}$ & $\mathbf{Z r}$ \\
\hline$\gamma$ phase & 54.6 & 5.1 & 9.9 & 19.8 & 3.1 & 2.1 & 2.3 & 2.5 & 0.6 & $\mathrm{NA}$ & NA & NA \\
\hline$\gamma^{\prime}$ phase & 70.7 & 1.5 & 5.1 & 3.6 & 0.3 & 0.9 & 6.0 & 10.6 & 1.4 & NA & $\mathrm{NA}$ & $\mathrm{NA}$ \\
\hline
\end{tabular}

Compression samples were machined out of the $\mathrm{AD} 730^{\mathrm{TM}}$ industrial billet. To trigger the recrystallization of the alloy and observe $\gamma-\gamma^{\prime}$ interactions, the samples were isothermally compressed below the $\gamma^{\prime}$ solvus (which is about $1,110^{\circ} \mathrm{C}$ ) and water quenched. Sample sections were ground and polished with first diamond suspensions down to $1 \mu \mathrm{m}$ then 0.02 $\mu \mathrm{m}$ colloidal silica (OPS) on a vibratory polishing machine. Hot compression led to a partially recrystallized microstructure exhibiting many microstructural features, including recrystallized/unrecrystallized grains and several $\gamma^{\prime}$ populations with characteristic sizes. Thus, this kind of microstructure was found relevant to discuss the performances of the iCHORD-SI combination.

\subsection{The iCHORD-SI combination}

The ion CHanneling Orientation Determination (iCHORD) technique has been recently developed by Langlois et al. [1] to obtain orientation maps using the ion channeling effect. Ion/matter interactions produce secondary electrons whose intensity depends on how deep the ions are channeled through the crystal, and so on the orientation of the crystallographic planes relative to the ion beam $[15,17,18]$. Then, if the crystal position changes relative to the ion beam, the intensity of the collected secondary electrons varies following an intensity profile which is characteristic of the crystallographic orientation of the crystal.

The experimental setup of the iCHORD technique (Figure 1.a) has been optimized so that each intensity profile corresponds to a unique crystallographic orientation. The sample is first tilted at a $40^{\circ}$ fixed angle around an axis $\left(\mathbf{e}_{\mathbf{y}}\right)$ which lies in its surface. Next, with a fixed azimuthal rotation step, the sample performs a complete rotation around its tilted normal $\left(\mathbf{e}_{\mathbf{z}}\right)$ to make secondary electron intensity vary (Figure 1.c). One Secondary Electron (SE) image is acquired at each azimuthal position. Then, using the Fiji image processing software [19], SE images undergo tilt correction, rotation correction, are aligned and cropped all together to get a stack of aligned images. The intensity of a given point of the scanned area varies as the stack is browsed, allowing the plot of a so-called intensity profile (i.e. intensity of the point in the SE image as a function of the azimuthal rotation angle, Figure 1.b). Finally, during the post-treatment stage, intensity profiles are compared to theoretical intensity profiles stored in a database, each theoretical intensity profile corresponding to a known crystallographic orientation. The building of the theoretical profiles and the database search algorithm are precisely described in [1]. The theoretical profile which matches the best with the experimental intensity profile gives the crystallographic orientation of the point.

The ion-matter interactions also create secondary ions whose intensity mainly depends on the atomic number of the excited atoms [15]. Thus, collected by a Secondary Ion (SI) detector, the Secondary Ion signal highlights the chemical contrast of the microstructure. In the present case, the $\gamma^{\prime}$ phase, which contains lighter elements (Table 2), appears much brighter than the $\gamma$ phase on SI images (Figure 1.d). To properly extract the $\gamma$ ' phase from SI data, several SI images have been acquired at different rotation angles and summed all together so as the channeling contrast of the SI images does not interfere with the chemical 
information. Finally, to separate the $\gamma^{\prime}$ precipitates from the $\gamma$ matrix an intensity threshold is applied to the sum image.

Using the TESCAN Lyra3 FIB-SEM of the Orsay Physics Company, combined iCHORD and SI scans have been performed on a sample section. The FIB was equipped with a Gallium ion source and the beam setup for data acquisition was $30 \mathrm{kV} / 48 \mathrm{pA}$. The pixel size was 36 $\mathrm{nm}$ and the azimuthal rotation step was $4^{\circ}$, which implied the acquisition of $90 \mathrm{SE}$ images to achieve the complete rotation of the sample. With the same $36 \mathrm{~nm}$ pixel size, four SI images were also acquired at different azimuthal rotation angles.
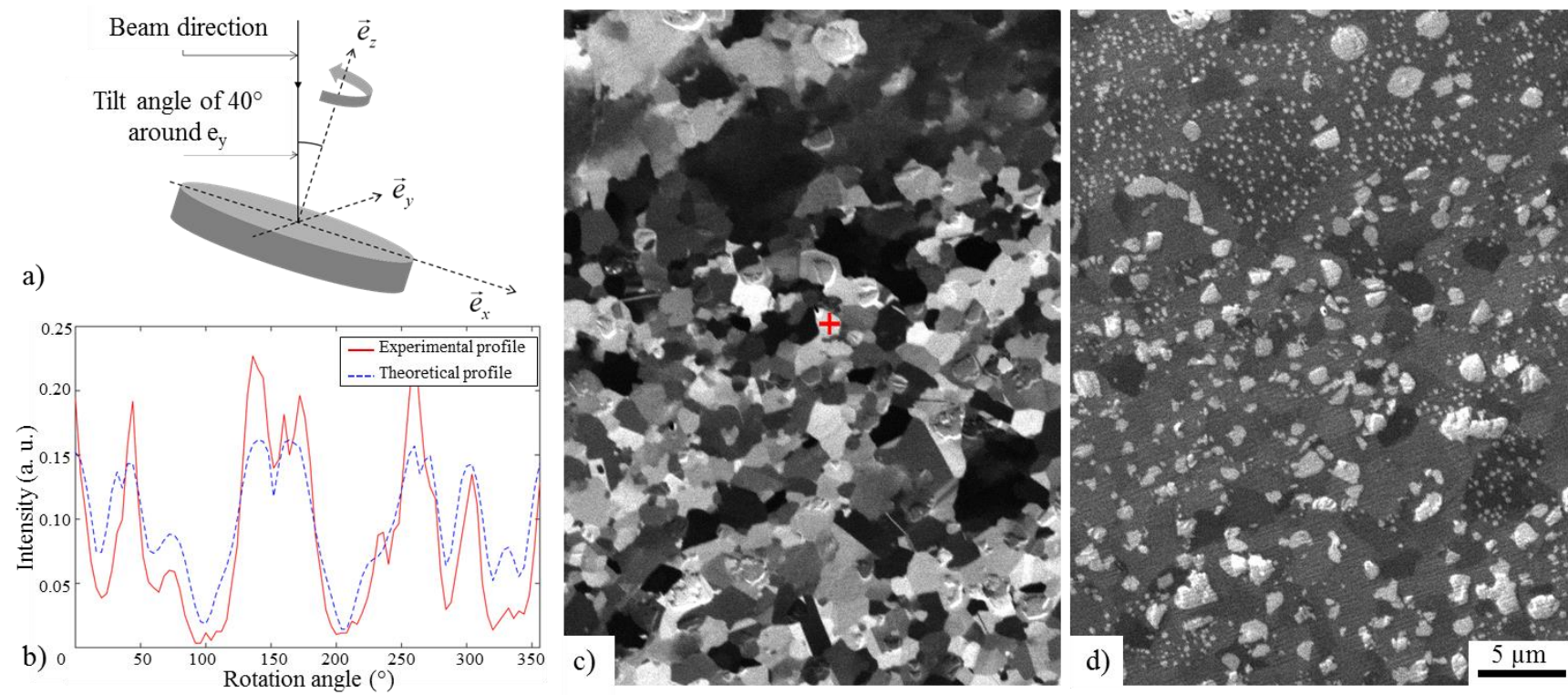

Figure 1. Principle of the iCHORD-SI combination. a) Experimental setup in the FIB microscope. b) Theoretical intensity profile (blue dashed line) matching with an experimental profile (red line) - data of the point highlighted by a red cross on c). c) Secondary Electron (SE) image for one azimuthal position around $e_{z}$ (the orientation contrast arises from the ion channeling effect). d) Secondary Ion (SI) image for the same azimuthal angle (gray levels mainly depend on the chemical composition even if a slight orientation contrast is also visible). Both SE and SI images are tilt corrected.

\subsection{EDS-EBSD coupling: example of the $\boldsymbol{\gamma}-\boldsymbol{\gamma}^{\prime}$ nickel-based superalloys}

The crystallographic lattice parameters of the $\gamma$ and $\gamma^{\prime}$ phases are very close to each other. However, theoretically EBSD patterns of both phases do exhibit differences. Indeed, the $\gamma^{\prime}$ phase has an ordered structure which allows crystallographic planes with both odd and even Miller indices to diffract, contrary to the $\gamma$ phase whose disordered structure leads to a systematic extinction for such planes. So, in principle, detecting the presence or the absence of the superstructure reflections should allow the distinction of the two phases with EBSD. But, the corresponding Kikuchi bands exhibit very low intensities compared to the others and would thus require the acquisition of very high signal-to-noise ratio EBSD patterns to be detected. This does not conform to the settings of commercial EBSD optimized for orientation mapping, which means a high indexing speed from noisy Kikuchi patterns (e.g. patterns of 160x120 pixels). Consequently, in practice the $\gamma$ and $\gamma^{\prime}$ grains of same crystallographic orientation display identical patterns (Figures 2.a, 2.b and 2.c) and the EBSD indexing software is unable to decide alone whether a given diffraction pattern arises from one phase or the other. This is why extra information is needed to distinguish the two phases in an EBSD map. 
The $\gamma$ and $\gamma^{\prime}$ phases can easily be identified with the EDS technique relying on their Nickel, Titanium and Chromium contents (Table 2, Figures 2.d, 2.e and 2.f). Thus, simultaneously to the EBSD scan, EDS spectra are acquired for each point of the map. Afterward, from EDS data, $\mathrm{Ni}, \mathrm{Ti}$ and $\mathrm{Cr}$ ratios are defined as references for each phase. The re-analysis of the EBSD data assigns to an indexed point the phase whose reference ratios are the closest to the $\mathrm{Ni}$, Ti and Cr ratios quantified in this point. Finally, an EBSD map with $\gamma / \gamma^{\prime}$ discrimination is obtained (Figure 2.g).

EDS-EBSD coupled acquisitions have been performed on a similar sample than that used for the iCHORD-SI acquisition, using the same $36 \mathrm{~nm}$ pixel size. Three accelerating voltages $10 \mathrm{kV}, 15 \mathrm{kV}$ and $20 \mathrm{kV}$ were tested corresponding to a beam current in the $7-10 \mathrm{nA}$ range. The EDS/EBSD system is a QUANTAX system from the Bruker Company composed of an EDS XFlash 5030 detector and an $\mathrm{e}^{-}$Flash $^{\mathrm{HR}}$ EBSD detector, controlled by the ESPRIT software package. The ESPRIT software package was used for both the acquisition and the re-analysis of the EBSD data with the EDS data (semi-automated procedure). This system is installed on a Field Emission Gun scanning electron microscope (Zeiss Supra 40).
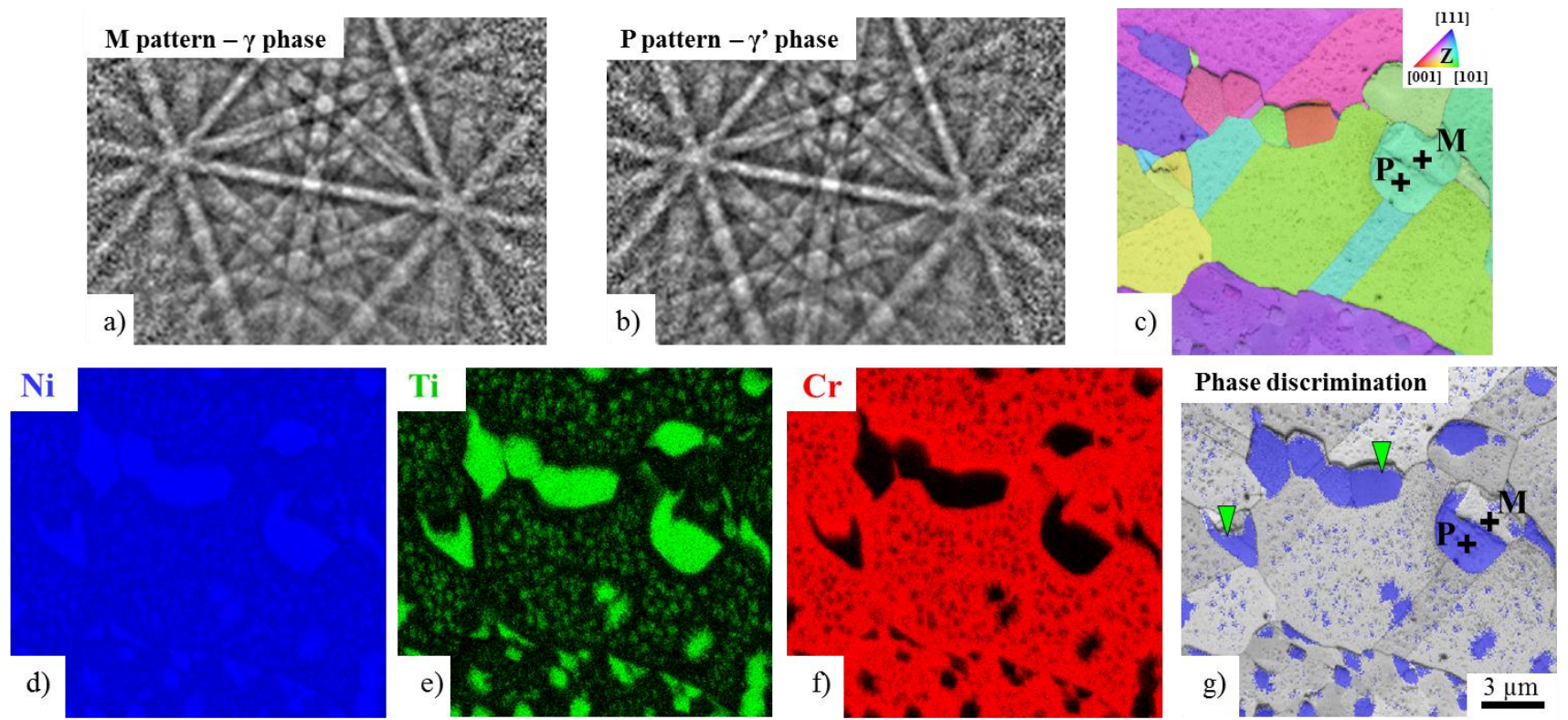

Figure 2. Principle of the EDS-EBSD coupling. a) and b) Kikuchi patterns (160x120 pixels) of the points displayed on c): $M$ (Matrix - $\gamma$ phase) and P (Precipitate - $\gamma$ ' phase). c) EBSD orientation map, the color code (standard triangle) displays the crystal direction parallel to the $Z$ axis, normal to the scanned area. d) to f) EDS elemental maps. g) $\gamma$ ' phase obtained from the EDS-EBSD coupling method highlighted blue on the EBSD pattern quality map.

\section{Results and Discussion}

\subsection{Raw data quality and applied filters}

Some of the results presented in the following parts were analyzed and displayed using the free MTEX Matlab toolbox [20].

During an EBSD scan, points located at grain boundaries show poor pattern quality because the diffraction patterns of the adjacent grains overlap. This explains why grain boundaries are non-indexed with the EBSD technique (Figure 3). 


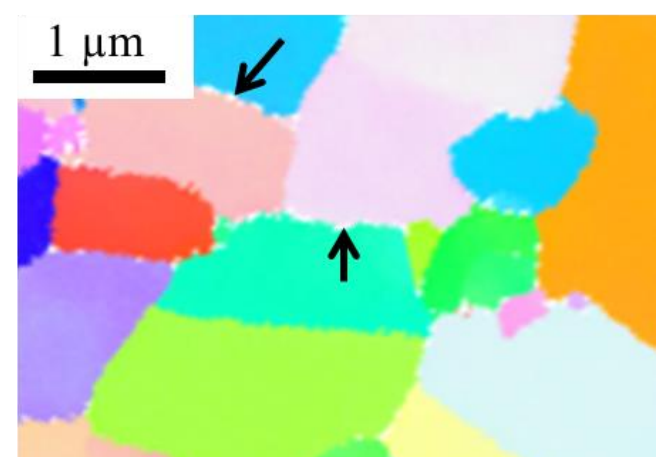

Figure 3. Grain boundaries with the EBSD technique. EBSD orientation map, non-indexed points plotted white. Black arrows highlight non-indexed points at grain boundaries.

On the other hand, with the iCHORD technique, all the points are indexed by the theoretical profile which suits the best. Then, to assess the quality of the correlation between the experimental and theoretical intensity profiles, a confidence index can be calculated. This confidence index may be defined by (1-ED) where ED means the Euclidian Distance between the two normalized profiles (this confidence index is plotted in Figure 12.a). By default, no threshold value is applied on the confidence index of the iCHORD raw dataset. That is why by default there is no zero-solution points and orientations are proposed even for points located at grain boundaries (Figures 4.e, 5.c and 6.a). This will be referred to as "grain boundary indexing" in the following.

"Grain boundary indexing" is actually an artifact of the iCHORD technique since most of the time the orientations assigned to grain boundaries are totally different from that of their surrounding grains (Figure 4.e). Those wrong orientations at grain boundaries can be explained by two effects resulting from the sample abrasion which occurs during the scan, even at beam currents as low as $48 \mathrm{pA}$. Indeed, between the first and the fourth SI image, 220 $\mathrm{nm}$ large precipitates have vanished (green circles on Figures 4.a and 4.b) and others have appeared. In the sample section, the largest diameter of the small precipitate population is approximatively $340 \mathrm{~nm}$ (Figure 4.a). This means that, if the small precipitates are initially spheres with a $340 \mathrm{~nm}$ diameter, at least the removal of a $40 \mathrm{~nm}$ layer is required to make the precipitates with a $220 \mathrm{~nm}$ diameter in the sample section disappear. The fourth SI image was acquired at one-third of the iCHORD experiment, so that the removed layer during the complete rotation is about $120 \mathrm{~nm}$.

The first effect of abrasion is boundary raveling (Figure 4). Abrasion depends on the phase - because the $\gamma$ and $\gamma$ ' phases do not have the same "hardness" - and depends also on crystal orientation within the same phase. That is why, after several FIB scans some $\gamma-\gamma$ ' and $\gamma-\gamma$ grain boundary planes are revealed and an extra topographic contrast is added in SE images (yellow arrows on Figure 4.c). In fact, when the revealed grain boundary plane is exposed (or hidden) to the SE detector, bright (or dark) saturation is observed. These alternative signal saturations along grain boundaries degrade the SE images alignment (Figure 4.d) and leads to thick grain boundaries. The pixels within these thick boundaries are indexed by the closest theoretical profile, which is likely to be different from that of the adjacent grains due to the alternative signal saturations (Figure 4.e), but which may nevertheless present a good fitting (Figure 4.f and 4.g).

The second effect of abrasion is boundary displacement (Figure 5). If a grain boundary plane is tilted regarding the sample surface, then the intersection of the grain boundary plane with the observed section (i.e. the grain boundary trace) will progressively move as the matter is removed from the sample surface by the ion beam (Figure 5.b). So, a point initially in a grain can belong to the neighbor grain at the end of the iCHORD scan. The intensity profile acquired in such a point begins by the intensity profile of the first grain it belonged and ends by that of the second grain (Figure 5.d). The whole profile is assigned to a theoretical profile 
which, once again, is likely to be different from that of the adjacent grains (Figure 5.c), but which may nevertheless present a good fitting.
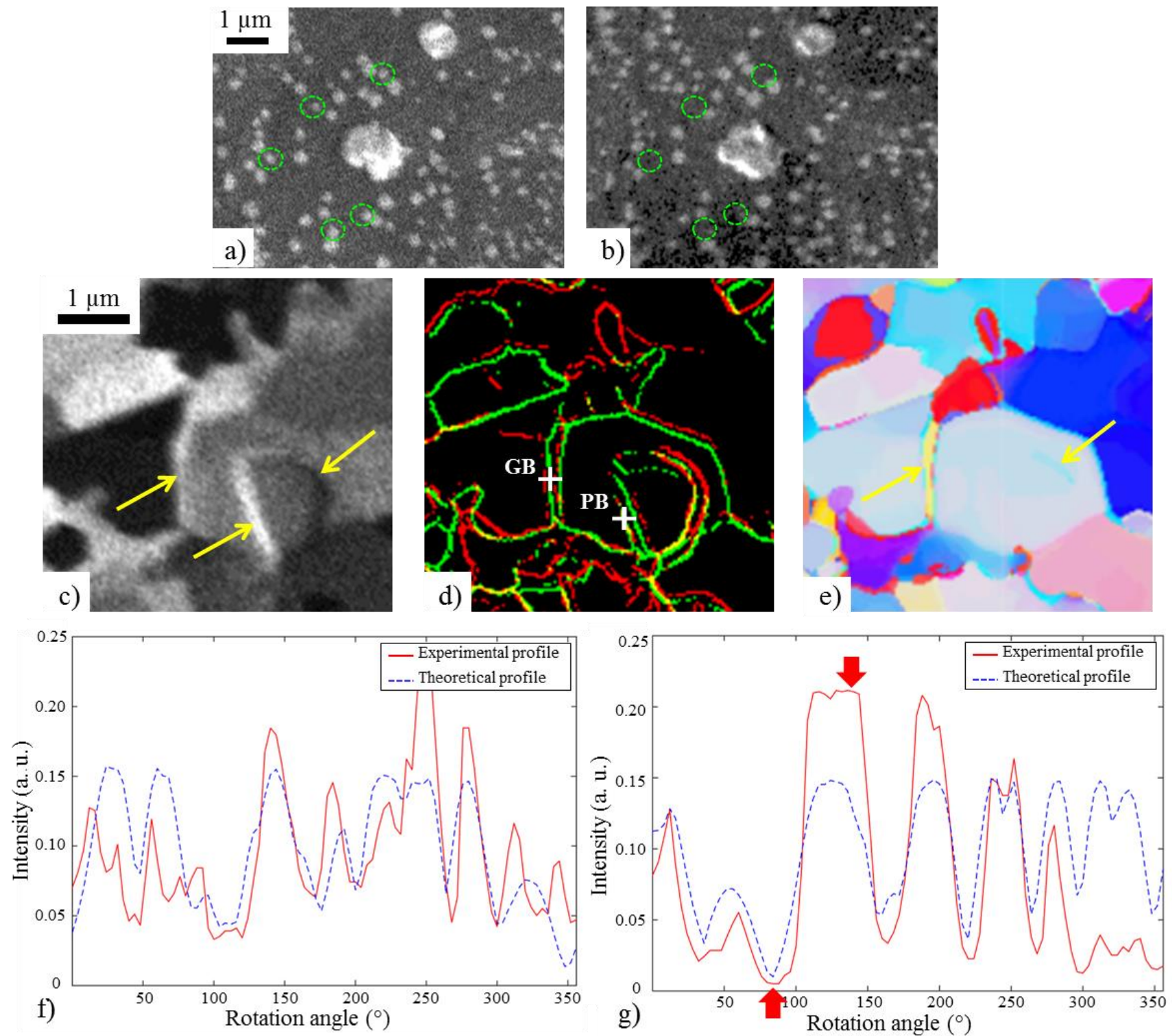

Figure 4. Boundary raveling resulting from FIB abrasion. a) First SI image acquired at the initial sample position. b) Fourth SI image acquired at one-third of the complete rotation. Green circles highlight small precipitates which have vanished between a) and b). c) SE image, yellow arrows showing boundary raveling due to abrasion. d) Grain boundaries obtained from SE images processing with Fiji [19]. Boundaries detected on the first ten SE images and boundaries detected on the last ten SE images plotted green and red respectively. e) iCHORD orientation map, yellow arrows showing raveling boundaries whose orientations are different from that of the neighboring grains. f) and $g$ ) Intensity profiles acquired in the grain boundary "GB" and precipitate boundary "PB" points displayed in d). Red arrows show signal saturation.

Thus, theoretical profiles can correctly fit profiles of points at grain boundaries by chance and this explains why the confidence index is not efficient to filter out the indexed grain boundary from the iCHORD raw dataset. So, another method was experimented: the microstructure edges were extracted by processing the SE images with the Fiji programs (Figure 6.b) and then stated as non-indexed points in the iCHORD dataset (Figure 6.d). This filtering is globally efficient but not perfect. Due to the FIB abrasion effects discussed above, 
the extracted edges can be larger than the "indexed interface". On the contrary, some small areas with inconsistent orientations still remain after filtering (black arrows Figure 6.d). Another idea to remove the "indexed boundaries" from the dataset could have been to: i) calculate the grains from the raw dataset, which would have identified artifact elongated grains along some real grain boundaries, ii) filter out those elongated grains based on a grain shape factor criterion. This idea was not investigated in this paper but may provide a finer treatment of the iCHORD raw data in the future. The following parts will present the results obtained from the iCHORD data filtered as explained above.

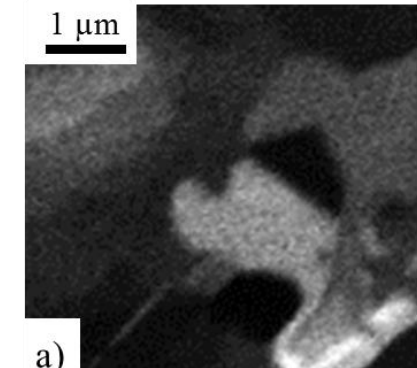

a)

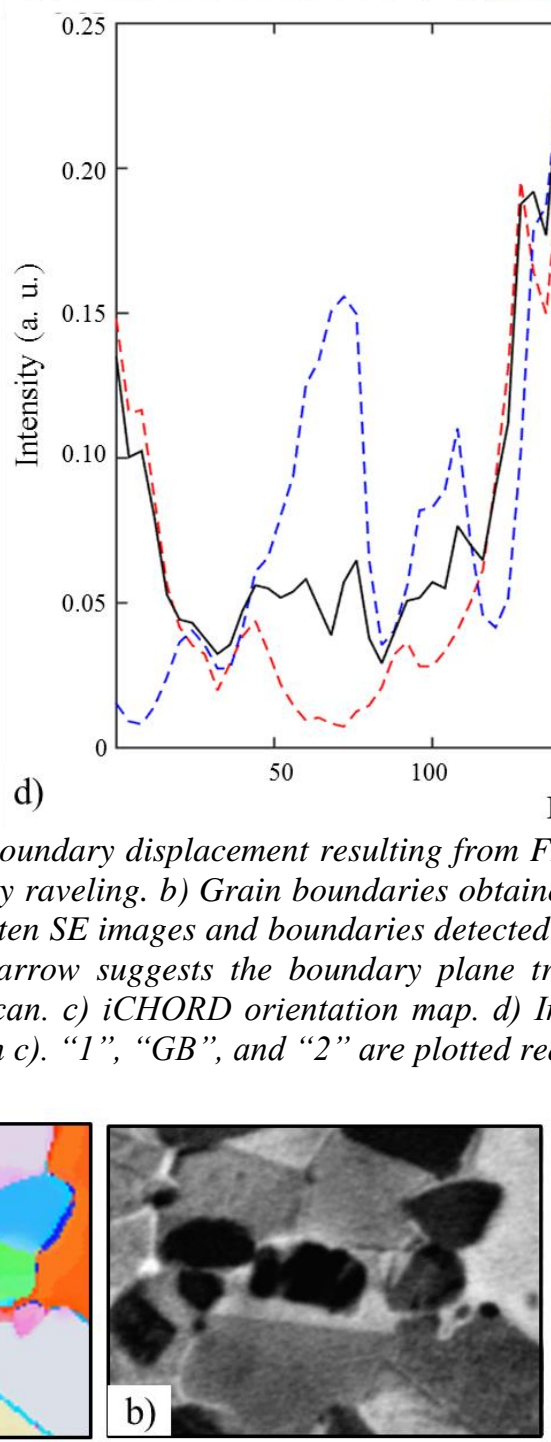

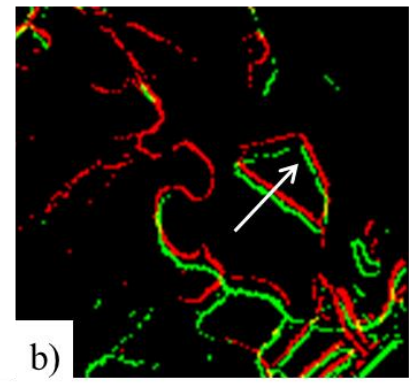

c)
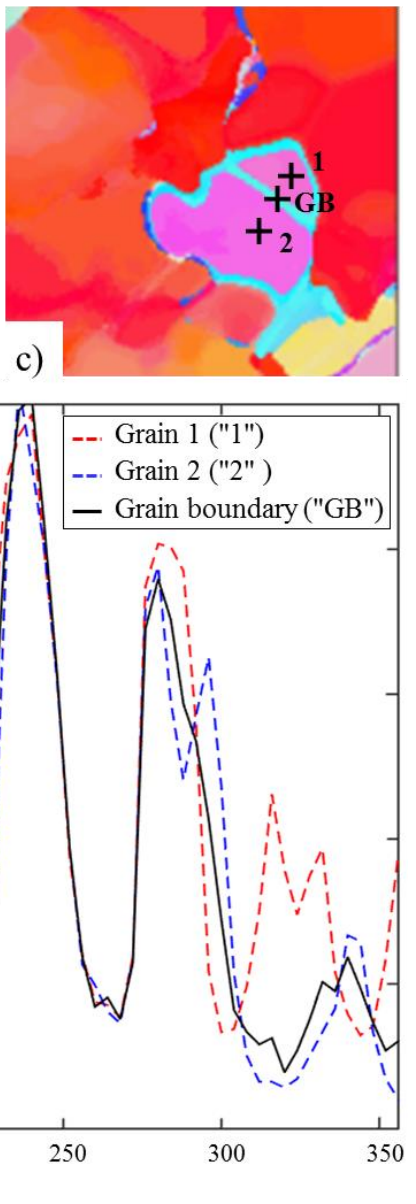

Figure 5. Boundary displacement resulting from FIB abrasion. a) SE image showing at the center a grain with no boundary raveling. b) Grain boundaries obtained from SE images processing with Fiji. Boundaries detected on the first ten SE images and boundaries detected on the last ten SE images plotted green and red respectively. The white arrow suggests the boundary plane trace has moved between the beginning and the end of the $i C H O R D$ scan. c) $i C H O R D$ orientation map. d) Intensity profiles acquired in the " 1 ", "GB" and " 2 " points displayed in c). "1", "GB", and " 2 " are plotted red, black and blue respectively.
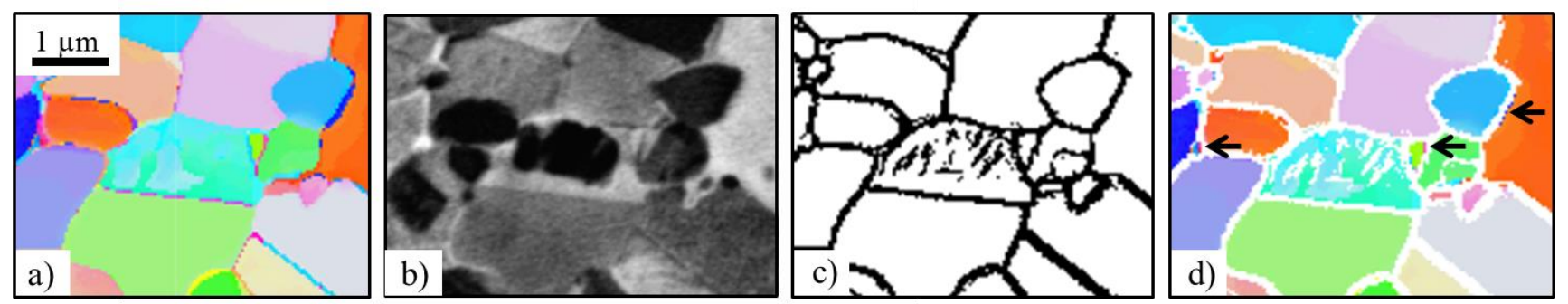

Figure 6. iCHORD data and applied filters. a) Orientation map from the raw iCHORD dataset, all the points are indexed by default. b) Backscattered Electron (BSE) image, showing that indexed boundaries do not correspond to real crystals but mainly to grain boundaries revealed by the ion beam. c) Edges of the iCHORD data extracted with the Fiji programs from the SE images. d) iCHORD orientation map after data filtering (i.e. where 
edges of the microstructure were stated as non-indexed points). Black arrows show remaining inconsistent orientations.

\subsection{Angular resolution}

In order to have a reference in term of crystal orientation, an EBSD scan at $20 \mathrm{kV}$ has been performed on the area chosen for the iCHORD-SI combination. The obtained iCHORD and EBSD maps are very similar, though some slight discrepancies are observed at some points (black arrows in Figure 7). These discrepancies can be explained by a change in the macroscopic reference frame - since the two experiments have been carried out in two different microscopes - but also by the different angular resolutions of the two techniques. Indeed, once the sample tilted the collected backscattered electrons and secondary ions come from volumes of approximately same sizes, that are typically within the $20-40 \mathrm{~nm}$ range [2123].
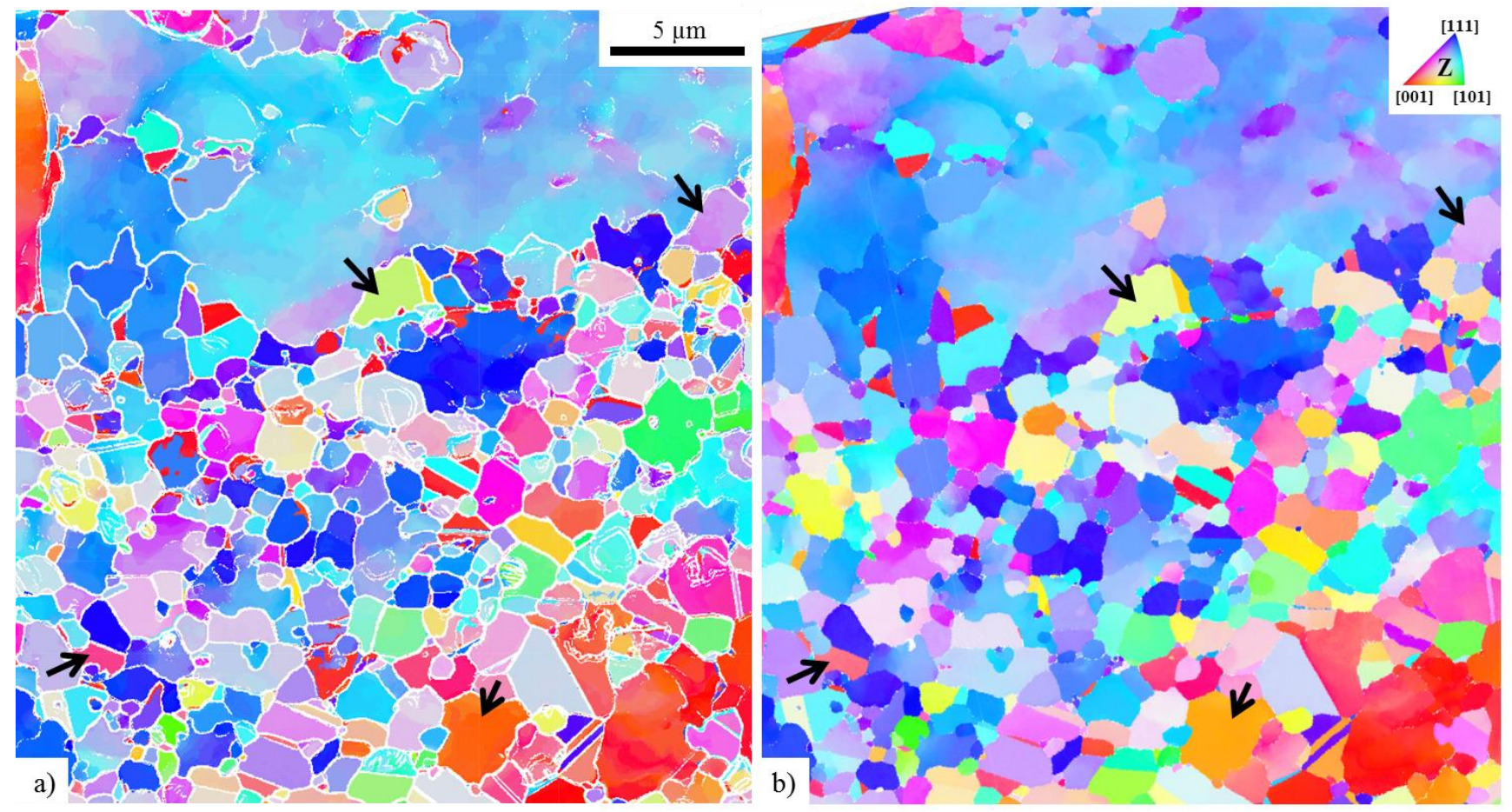

Figure 7. Orientations maps given by the iCHORD and EBSD techniques. The color code (standard triangle) displays the crystal direction parallel to the $Z$ axis, normal to the scanned area. a) iCHORD orientation map. $b$ ) EBSD orientation map. Black arrows highlight some differences between the iCHORD and EBSD maps.

To decorrelate orientation variations due to a change in the macroscopic reference frame (used to define the Euler angles) from that due to the angular resolution of the techniques, intragranular misorientation has been analyzed. Grains have been defined as groups of pixels with a neighbor-to-neighbor misorientation angle lower or equal to $10^{\circ}$, and misorientation to the mean grain orientation values have been calculated. Based on the misorientation to the mean grain orientation, the deformed grains can be easily distinguished from the newly recrystallized ones on both the EBSD and iCHORD maps (Figure 8.a and 8.b). Deformed grains have a high orientation spread because of lattice distortions induced by deformation. On the contrary, recrystallized grains have a very homogeneous orientation and have theoretically no deviation of individual pixel orientation from the mean grain orientation. Thus, the misorientation measured between neighboring pixels in a recrystallized grain is representative of the angular resolution of the technique. This way, from the orientation variations observed in Figure 8.c and 8.d, the angular resolution is estimated to be about $0.75^{\circ}$ 
for EBSD and a bit less than $1.5^{\circ}$ for iCHORD. This difference in angular resolution makes that the $10^{\circ}$ grain threshold does not lead exactly to the same grains for the iCHORD and EBSD datasets, and this accounts for the differences in misorientation to mean orientation values observed between Figures 8.a and 8.b. However, the misorientation profiles plotted in Figure 8.e and 8.f demonstrate that even if small discontinuities $\left(<1.5^{\circ}\right)$ are very roughly captured by the iCHORD technique due its angular resolution, similar grain substructures are globally revealed by both techniques. 

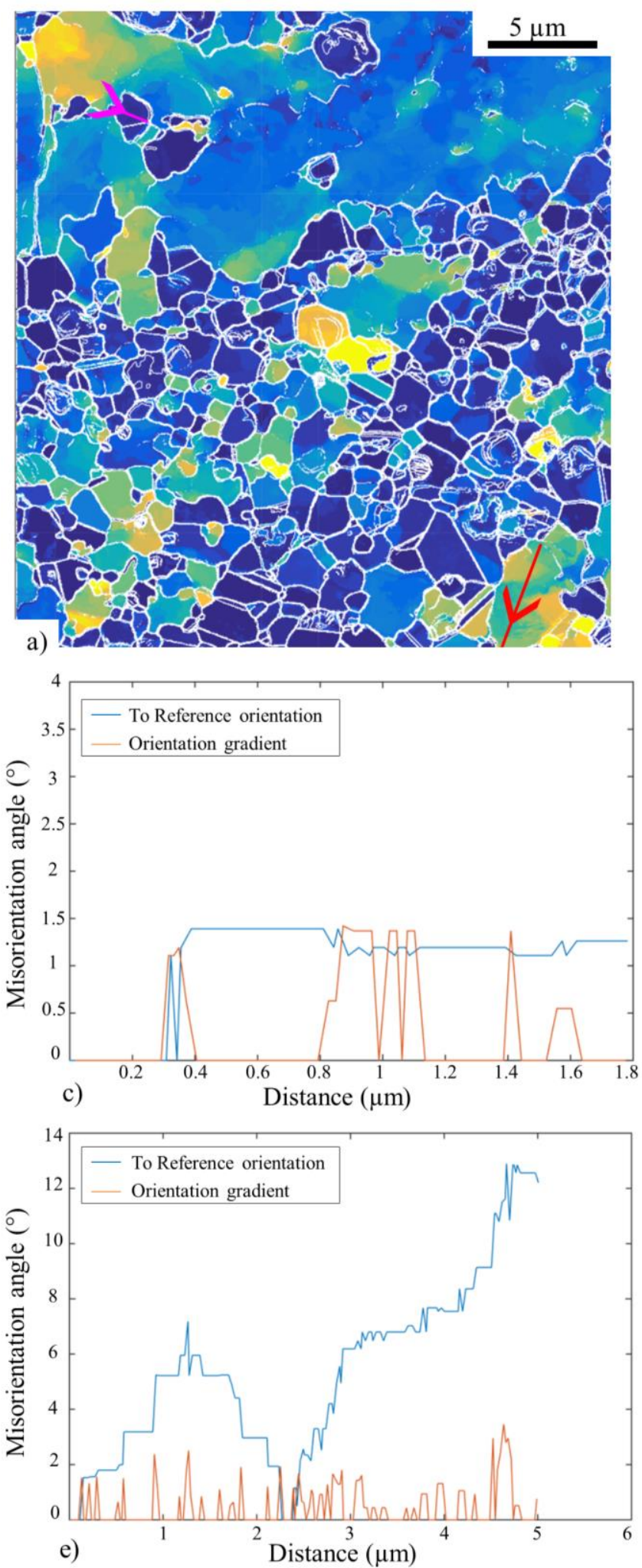
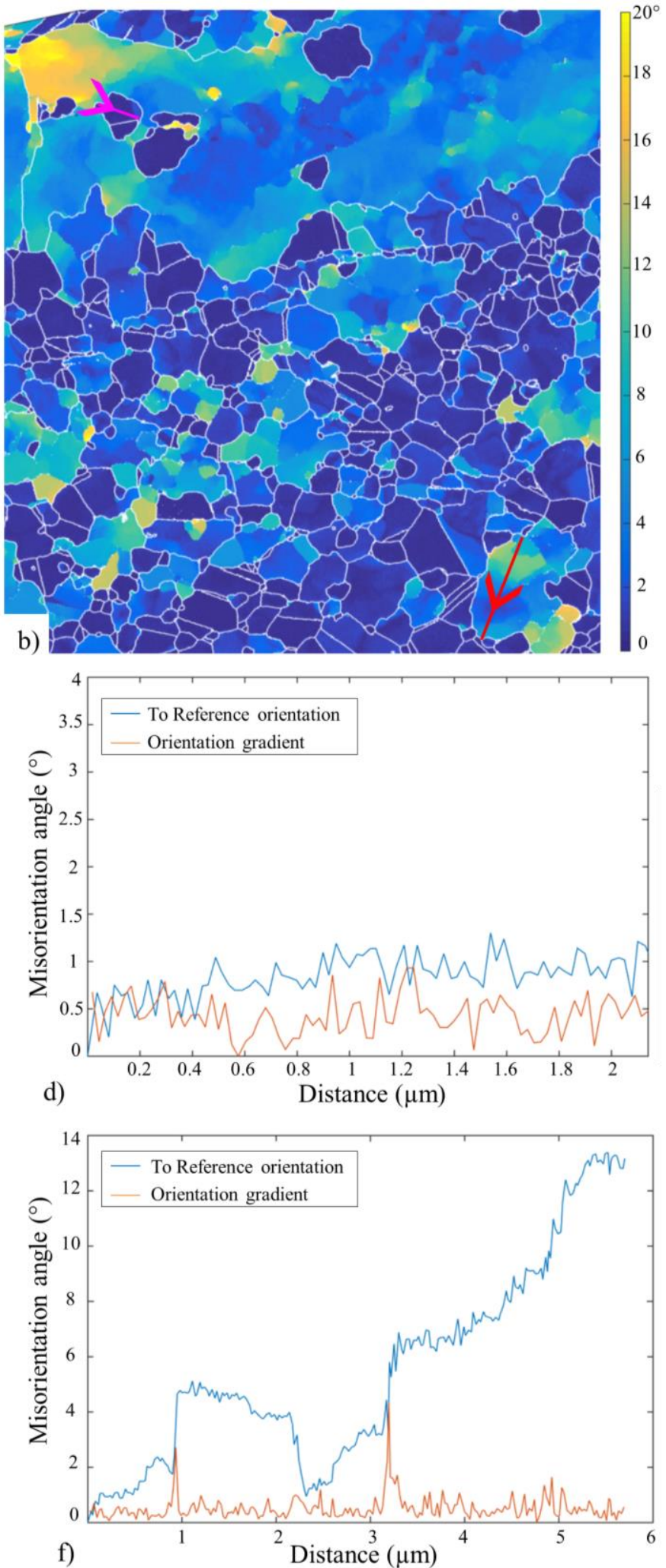

Figure 8. Angular resolution of the iCHORD and EBSD techniques. a) Misorientation to mean grain orientation map calculated from the iCHORD dataset (misorientation angle threshold: $10^{\circ}$ ), grain boundaries plotted white. b) Misorientation to mean grain orientation map calculated from the EBSD dataset (misorientation angle threshold: $10^{\circ}$ ) grain boundaries plotted white. c) and d) Misorientation profile along the pink line in the recrystallized grain shown in a) and b) for the iCHORD and EBSD techniques respectively. e) and f) Misorientation profile along the red line in the non-recrystallized area shown in a) and b) for the ICHORD and EBSD techniques respectively. 


\subsection{Spatial resolution of the phase discrimination}

Figure 9 shows that the SI images can resolve the $\gamma^{\prime}$ precipitates down to $150 \mathrm{~nm}$, which means all the precipitates of the studied microstructure including the smallest ones judging from the BSE image in Figure 9.a (the differences between the BSE and SI image arise from the ion beam erosion which occurred between the first SI image and the BSE image acquired after the iCHORD scan). The resolution of the SI images is related to the volume from which the collected secondary ions come: typically some tens of nanometers [22,24].
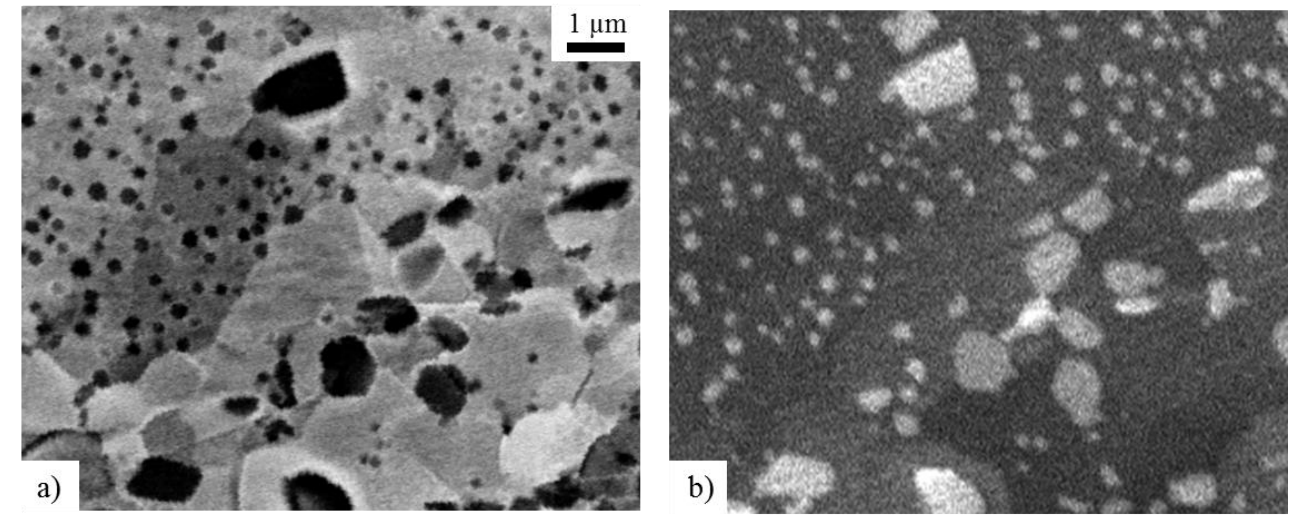

Figure 9. $\gamma^{\prime}$ phase from the raw SI signal, comparison with the BSE signal. a) BSE image acquired after the iCHORD-SI scan at $0^{\circ}$ tilt. b) First SI image of the iCHORD-SI scan.

To evaluate the performance of the SI signal, three EDS-EBSD maps have been acquired at $10 \mathrm{kV}, 15 \mathrm{kV}$ and $20 \mathrm{kV}$ on an area where precipitate sizes were similar to that analyzed with the SI signal. For each EDS-EBSD map, the exposure time per pixel was adapted to get an X-ray spectrum with 3,000 counts for each point of the map. Indeed, 2,000 to 3,000 counts per spectrum are empirical values leading to a signal-to-noise ratio large enough for a semiquantitative analysis of the EDS spectrum. With Figure 10 one can check that for the chosen exposure times, the quality of the diffraction patterns was sufficient to perform EBSD, even at $10 \mathrm{kV}$ which is a quite low accelerating voltage for the technique.

The chemical contrast resulting from the three EDS scans is presented in Figure 11. As expected, the higher is the accelerating voltage, the lower the $\gamma^{\prime}$ precipitates are resolved. Indeed, if the X-ray absorption in the sample is neglected, for a $0^{\circ}$ tilted sample the maximum escape depth of the X-rays can be estimated using the formula proposed by Anderson and Hasler [13,25]: $X_{e} \approx \frac{0.064}{\rho} \cdot\left(E_{0}^{1.68}-E_{C}{ }^{1.68}\right)$, where $X_{e}$ is the average maximum escape depth of the X-rays $(\mu \mathrm{m}), \rho$ is the density of the material $\left(\mathrm{g} . \mathrm{cm}^{-3}\right), E_{0}$ the incident electron beam energy $(\mathrm{keV})$ and $E_{C}$ the critical excitation energy $(\mathrm{keV})$. Considering approximate atomic compositions for the $\gamma$ and $\gamma^{\prime}$ phases, ranges for the maximum escape depth at a $0^{\circ}$ tilt are given in Table 3. Tilting the sample at $70^{\circ}$ impacts the shape of the $\mathrm{X}$-ray emission volume regarding the sample surface [26]. This implies that the maximum escape depth is in fact a bit smaller for a $70^{\circ}$ tilt than for a $0^{\circ}$ tilt. Nevertheless, Figure 11 shows that for a $70^{\circ}$ tilt EDS at $10 \mathrm{kV}$ is the only appropriate configuration to properly resolve the $150-340 \mathrm{~nm}$ precipitates (although the results are a bit affected by the sample relief). In addition, as the volume from which the collected X-ray arise is deeper than that of the backscattered electrons, once the sample tilted at $70^{\circ}$ EDS data are slightly shifted compared to EBSD data [26]. The shift is highlighted by green arrows in Figure 1. This problem does not exist with the iCHORD-SI combination since SE and SI emission volumes have nearly the same sizes. 

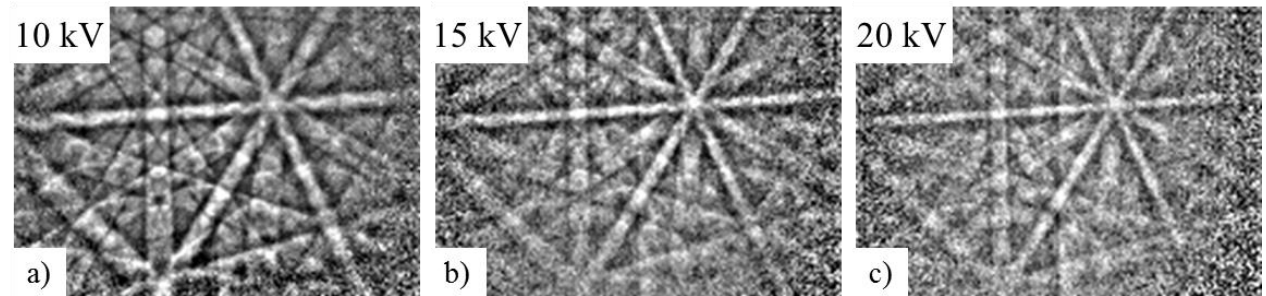

Figure 10. EBSD pattern for the different EDS-EBSD settings-160x120 pixels patterns. a) EDS-EBSD coupling at $10 \mathrm{kV}$. b) EDS-EBSD coupling at $15 \mathrm{kV}$. c) EDS-EBSD coupling at $20 \mathrm{kV}$.
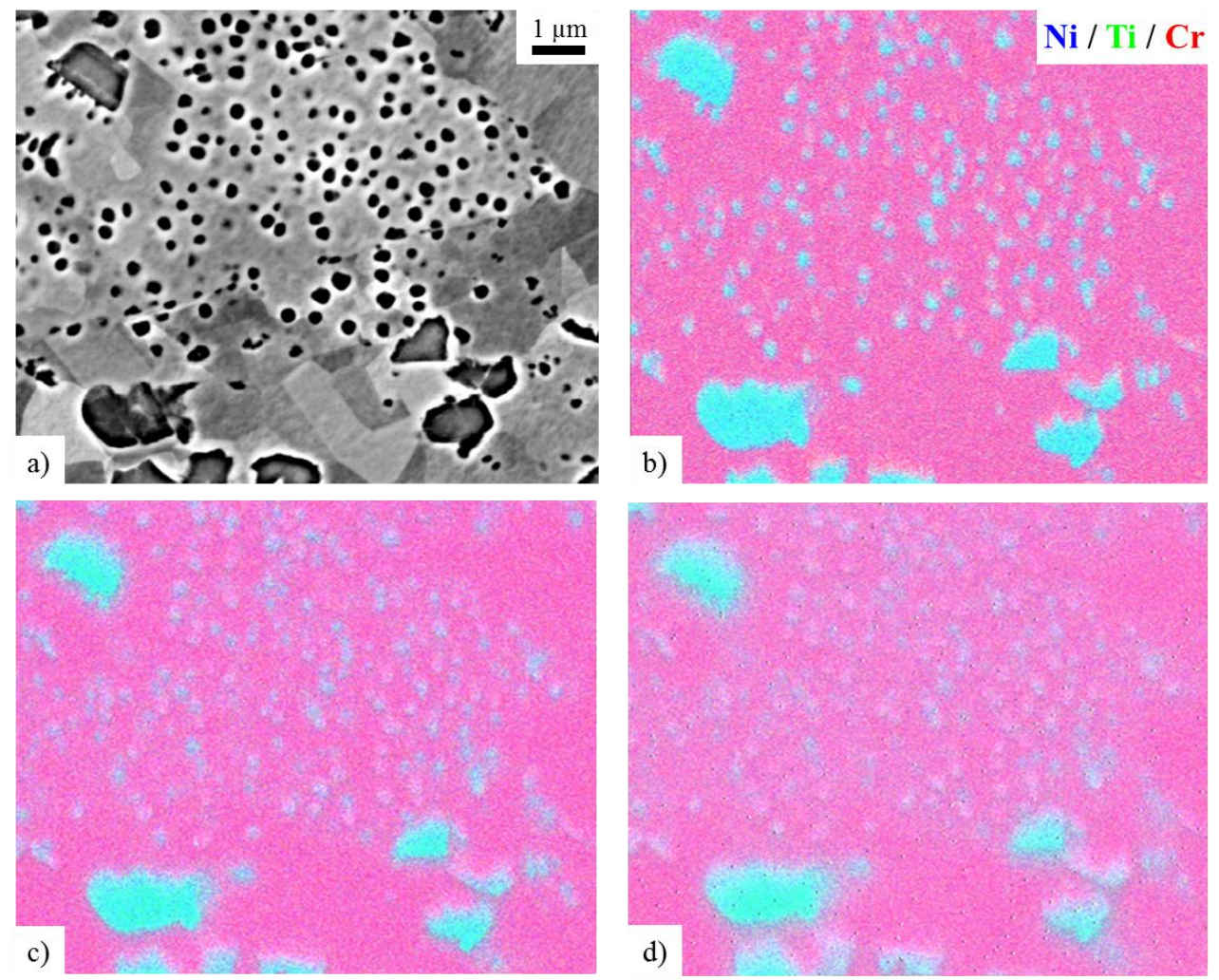

Figure 11. $\gamma$ ' phase from the raw EDS signal, comparison with the BSE signal. a) BSE image, $\gamma$ ' phase appears dark because of its content in light elements and the sample relief. b), c) and d) Overlay of the $\mathrm{Ni}$, Ti and $\mathrm{Cr}$ elemental maps obtained at $10 \mathrm{kV}, 15 \mathrm{kV}$ and $20 \mathrm{kV}$ respectively. $\mathrm{Ni}$, Ti and Cr elemental maps are plotted blue, green and red which reveals the $\gamma$ ' phase as light blue.

Table 3. Estimation of the maximum $X$-ray escape depth at a $0^{\circ}$ tilt using Anderson and Hasler's formula.

\begin{tabular}{|c|c|c|c|c|c|c|c|}
\hline \multirow[b]{2}{*}{ Phase } & \multirow[b]{2}{*}{$\begin{array}{l}\text { Density } \\
\left({\left.\mathrm{g} . \mathrm{cm}^{-3}\right)}^{-}\right.\end{array}$} & \multicolumn{2}{|c|}{$10 \mathrm{kV}$} & \multicolumn{2}{|c|}{$15 \mathrm{kV}$} & \multicolumn{2}{|c|}{$20 \mathrm{kV}$} \\
\hline & & $E_{C}(\mathrm{keV})$ & $X_{e}(\mu \mathrm{m})$ & $E_{C}(\mathrm{keV})$ & $X_{e}(\mu \mathrm{m})$ & $E_{C}(\mathrm{keV})$ & $X_{e}(\mu \mathrm{m})$ \\
\hline $\begin{array}{c}\boldsymbol{\gamma} \\
\sim \mathrm{Ni}-22 \% \mathrm{Cr}-12 \% \mathrm{Co}-6 \% \mathrm{Fe}-4 \% \mathrm{Al} \\
\end{array}$ & 8.22 & \multirow[t]{2}{*}{$\begin{array}{c}0-5.987 \\
(\mathrm{Cr}-\mathrm{K})\end{array}$} & $0.22-0.37$ & \multirow[t]{2}{*}{$\begin{array}{c}0-8.339 \\
(\mathrm{Ni}-\mathrm{K})\end{array}$} & $0.46-0.74$ & \multirow[t]{2}{*}{$\begin{array}{c}0-8.339 \\
(\mathrm{Ni}-\mathrm{K})\end{array}$} & $0.92-1.19$ \\
\hline $\begin{array}{c}\gamma^{\prime} \\
\sim \mathrm{Ni}-12,5 \% \mathrm{Ti}-12,5 \% \mathrm{Al}\end{array}$ & 7,58 & & $0.23-0.40$ & & $0.50-0.80$ & & $1.00-1.29$ \\
\hline
\end{tabular}

The $\gamma^{\prime}$ phase discriminations obtained from the processing of the iCHORD-SI and EDSEBSD data are presented in Figure 12. The resolution of the iCHORD-SI map (Figure 12.a) is slightly degraded compared to that of the original SI images (e.g. Figure 9.b). Indeed, the evolution of the precipitates resulting from the abrasion of the sample surface as well as the treatment applied to SI data (alignment, sum and threshold adjustment) are responsible for the slight degradation. Regarding the EDS-EBSD method, as implemented in the ESPRIT software, the $\gamma^{\prime}$ phase discrimination (Figure 12.b-d) is not done for the non-indexed points 
since no crystallographic structure is assigned to the non-indexed points. Yet the non-indexed points appear more numerous for low accelerating voltages (e.g. $10 \mathrm{kV}$ ). Moreover, the $\mathrm{Ni}, \mathrm{Ti}$ and $\mathrm{Cr}$ ratios defined for Figure 12.b-d seem to be more adapted to the large precipitates than to the small ones which are consequently less resolved by the phase discrimination. In fact, the large and small precipitates do not form at the same temperature during the forming process, and thus may have somewhat different chemical compositions. But, above all, the resolution of small precipitates is subjected to the so-called "matrix effect" (i.e. the affection of the measured precipitate composition by that of the matrix located below), which goes worse and worse as the accelerating voltage increases due to the interaction volume. Different routes could be possible to improve the spatial resolution of the final EDS-EBSD discrimination, for instance: the adaptation of the composition ratios for each precipitate size, or the correction of the matrix effect. However, this would require EDS spectra with much higher signal-to-noise ratio and so the sacrifice of the acquisition speed.
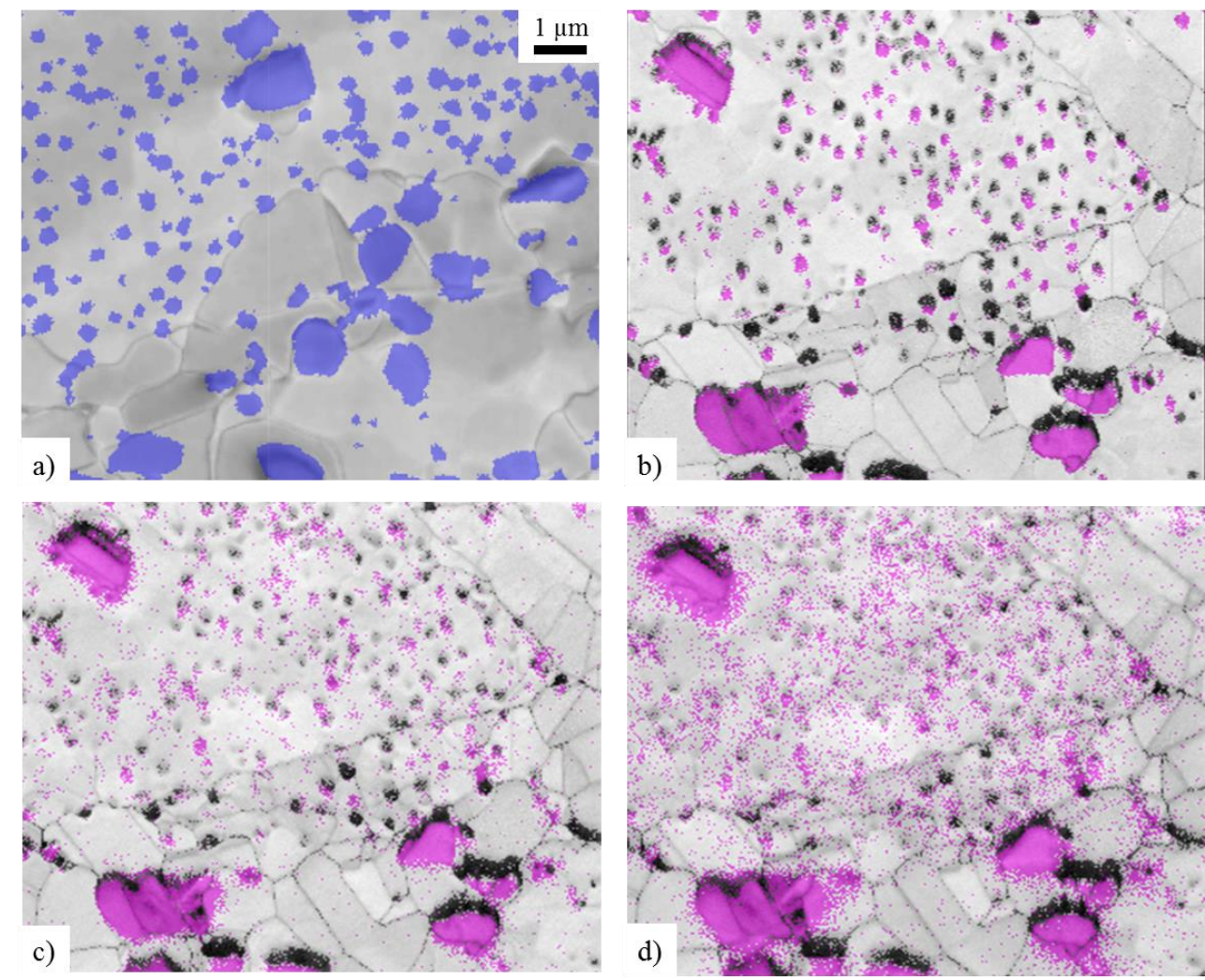

Figure 12. $\gamma$ ' phase discrimination obtained after SI and EDS-EBSD data processing. a) $\gamma$ ' phase resulting from the SI data overlaid on the iCHORD confidence index map. b), c) and d) $\gamma$ ' phase resulting from the EDS-EBSD data overlaid on the pattern quality map, and corresponding to the EDS-EBSD acquisitions at $10 \mathrm{kV}, 15 \mathrm{kV}$ and $20 \mathrm{kV}$ respectively. Non indexed points are displayed black.

\subsection{Acquisition and processing times}

As said above, for each EDS-EBSD map performed for the study the exposure time per pixel has been adapted so as to cumulate 3,000 counts per point. Consequently, acquisition time must increase as the accelerating voltage decreases (Table 4). The time given in the "Processing time / pixel" column corresponds to the time required by the ESPRIT software to re-analyze the EBSD data considering the EDS data with the defined $\mathrm{Ni}$, Ti and $\mathrm{Cr}$ reference ratios.

For the iCHORD-SI acquisition, relatively low scanning speeds were chosen to get a good signal-to-noise ratio while limiting the sample abrasion by the ion beam. The chosen scanning speeds lead to $0.010 \mathrm{~ms} / \mathrm{pixel}$ and $0.032 \mathrm{~ms} / \mathrm{pixel}$ for the SE and SI images respectively (Table 
4). These times do not include the time required for the stage rotation which may be automated. The time given in the "Processing time / pixel" column corresponds to the average time the iCHORD algorithm takes to assign a theoretical profile to an experimental intensity profile. However, with the iCHORD technique, the number of acquired points is twice bigger than the number of effectively analyzed points. In fact, the analyzed area must be an area common to all the SE images after their alignment. Yet, SE images are square images acquired at different rotation angles and this makes the corners of the images unusable after the alignment. This explains why the effective analyzed area is much smaller than the original image size. This drawback could be fixed in the future by controlling the scan coils of the microscope to automatically perform a scan rotation at each stage rotation step.

Table 4. Acquisition and processing times for each method.

\begin{tabular}{|c|c|c|c|c|c|}
\hline Method & Settings & $\begin{array}{l}\text { Acquisition time } \\
\text { / pixel }\end{array}$ & $\begin{array}{l}\text { Pixels acquired } \\
\text { / Pixels analyzed }\end{array}$ & $\begin{array}{c}\text { Processing time / } \\
\text { pixel }\end{array}$ & "Total time" / pixel \\
\hline \multirow[t]{3}{*}{ EDS-EBSD } & $\begin{array}{l}10 \mathrm{kV} \\
(42 \mathrm{kCts})\end{array}$ & $36 \mathrm{~ms} \times 2$ frames & 1 & $1 \mathrm{~ms}$ & $36 \times 2+1=73 \mathrm{~ms}$ \\
\hline & $\begin{array}{l}15 \mathrm{kV} \\
(100 \mathrm{kCts})\end{array}$ & $15 \mathrm{~ms}$ x 2 frames & 1 & $1 \mathrm{~ms}$ & $15 \times 2+1=31 \mathrm{~ms}$ \\
\hline & $\begin{array}{l}20 \mathrm{kV} \\
(182 \mathrm{kCts})\end{array}$ & $8.3 \mathrm{~ms} \times 2$ frames & 1 & $1 \mathrm{~ms}$ & $8.3 \times 2+1 \approx 18 \mathrm{~ms}$ \\
\hline iCHORD-SI & $30 \mathrm{kV} / 48 \mathrm{pA}$ & $\begin{array}{c}\text { - SE image : } 0.010 \mathrm{~ms} \\
\text { x } 90 \text { images } \\
\text { - SI image : } 0.032 \mathrm{~ms} \\
\text { x } 4 \text { images }\end{array}$ & 2 & $17 \mathrm{~ms}$ & $\begin{array}{c}(0.010 \times 90+0.032 \times 4) \times 2+ \\
17 \approx 19 \mathrm{~ms}\end{array}$ \\
\hline
\end{tabular}

From total times approximatively spent per pixel reported in Table 4, it appears that performing the iCHORD-SI method is equivalent to perform an EDS-EBSD coupling at 20 $\mathrm{kV}$ (taking into account that the acquired points are twice numerous than that effectively analyzed ones).

\subsection{Discussion}

EBSD patterns give essential details on the crystallography of the phases and on the crystal orientations, with an angular resolution typically in the range of $0.5^{\circ}$. On the other hand, the current version of the iCHORD technique is adapted to get a good overview of the microstructure of a material with a know crystallographic structure: the $1.5^{\circ}$ angular resolution is enough to study the grain sizes and orientations as well as the recrystallization state of the microstructure.

Regarding the EDS technique, EDS spectra bring more than a chemical contrast as SI images do and so enables a semi-quantitative analysis of the chemical composition of the phases. Thus, numerous possibilities for the re-analysis of the EBSD data combined with the EDS spectra are possible afterward.Nevertheless, the spatial resolution of the EDS technique strongly depends on the accelerating voltage: the smaller is the accelerating voltage, the better is the spatial resolution. But decreasing the accelerating voltage must be compensated by a significant increase in the exposure time, in order to have a signal-to-noise ratio good enough for a semi-quantitative analysis of the spectra (about $30 \mathrm{~ms} \times 2$ for an EDS at $10 \mathrm{kV}$ ). By resolving $\gamma^{\prime}$ precipitates as small as $150 \mathrm{~nm}$, SI images have provided a spatial resolution as good as the EDS acquisition performed at $10 \mathrm{kV}$. But the total time per pixel is approximately three times smaller for the iCHORD-SI combination at $30 \mathrm{kV}$ than for the EDS-EBSD at $10 \mathrm{kV}$. Indeed, the exposure time represents a very large part of the total time spent for an EDS-EBSD map. Besides, long exposure times such as that applied at $10 \mathrm{kV}$ make the 
electron beam drift more significant during the EDS-EBSD maps. On the other hand, for the iCHORD-SI combination, the time dedicated to the data acquisition (SE and SI images) is very short compared to the time needed to process the data (attribution of a theoretical profile to the experimental intensity profile). This makes the time spent on the microscope much smaller for an iCHORD-SI map than for an EDS-EBSD map, whatever the accelerating voltage used for the EDS-EBSD acquisition. In addition, the different escape depths of the BSE and X-ray signals leads to a slight misalignment of the EBSD and EDS data because the sample is tilted at $70^{\circ}$. This does not occur for the iCHORD-SI data combination since SE and SI have arise from volume with approximately the same size.

But, the main drawback of the iCHORD-SI combination is the abrasion of the sample surface by the ion beam. Abrasion leads to boundary raveling and boundary displacement but also to the potential loss of the smallest grains and precipitates of the microstructure during the scan. This makes the iCHORD-SI combination a quite destructive technique which cannot be performed twice on exactly the same area. However, two ideas can be proposed to make the technique less destructive than what presented in the present paper. First, the number of acquired SE images can be decreased: it has been shown in [1] that $30 \mathrm{SE}$ images with a $12^{\circ}$ rotation step allow a correct description of the microstructure. Second, the scanning speeds could have been increased and denoising algorithms used to enhance the signal-to-noise ratio of the acquired images.

Thus, like the EDS-EBSD coupling, the iCHORD-SI combination has disadvantages and advantages. But by resolving precipitates down to $150 \mathrm{~nm}$ on an orientation map within a very reasonable time, this method is really interesting for the $\gamma / \gamma^{\prime}$ microstructures which displays a multimodal distribution of $\gamma^{\prime}$ precipitates from few micrometers to some tens of nanometers. Yet, for the study of polycrystals, a technique performed within a reasonable time is of particular interest since it allows a more statistical analysis of the microstructure.

Finally, Figure 13 shows the complete result of the iCHORD-SI combination, that is the $\gamma$ ' phase discriminated from the SI data overlaid on the orientation map obtained with the iCHORD technique. Black and white arrows prove the interest of the phase discrimination for the studied microstructure by highlighting two metallurgical phenomena based on the $\gamma^{\prime}$ precipitation: the so-called Smith-Zener pinning [6] enforced by precipitates as small as 150$340 \mathrm{~nm}$ on the grain boundaries and the heteroepitaxial recrystallization which starts by the nucleation of a coherent $\gamma$ shell around a $\gamma^{\prime}$ precipitate $[12,27]$. 


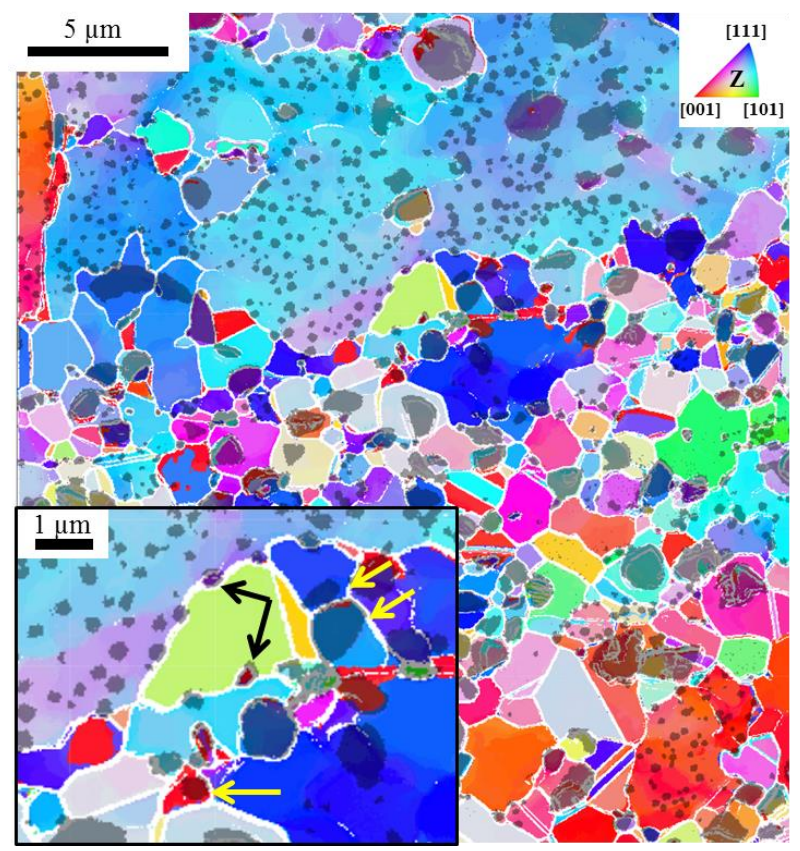

Figure 13. Results of the iCHORD-SI combination. The $\gamma$ ' phase is plotted dark grey and overlaid on the orientation map. Black and yellow arrows highlight grain boundary pinning and heteroepitaxial recrystallization respectively.

\section{Conclusions}

Because the $\gamma$ and $\gamma^{\prime}$ phases cannot be identified by the EBSD technique alone, other methods are needed to characterize the $\gamma-\gamma$ ' microstructures.

The EDS-EBSD coupling uses the chemical information provided by the EDS technique to discriminate the $\gamma$ and $\gamma^{\prime}$ phases on an EBSD map. The iCHORD-SI combination, which is an original method performed with a FIB microscope is based on the same idea: the iCHORD technique, recently developed by Langlois et al. [1], provides the crystallographic orientations based on the ion channeling contrast on Secondary Electron (SE) images while the chemical contrast of the Secondary Ion (SI) images is used to identify the $\gamma^{\prime}$ phase.

The performances of the iCHORD-SI combination on a partially recrystallized $\gamma-\gamma$ ' microstructure have been assessed using the EDS-EBSD coupling as a reference. The conclusions are the following:

- By default the iCHORD technique indexes each point of the map with the theoretical profile which suits the best to collected intensity profile. Orientations indexed at grain boundaries are most of the time inconsistent and result from boundary raveling and/or progressive boundary displacement induced by the ion beam. Thus, iCHORD data have to be treated to erase from the dataset the false orientations attributed to pixels on/along grain boundaries.

- The angular resolution of the iCHORD technique (about $1.5^{\circ}$ ) is a bit less good than that of the EBSD technique (about $0.50-0.75^{\circ}$ ). Nevertheless, this angular resolution is sufficient to succeed in catching most of the grain substructures.

- Unlike EDS and EBSD data, no misalignment between the SE and SI data are observed since secondary electrons and secondary ions come from volumes of similar sizes which are typically in the $20-40 \mathrm{~nm}$ range.

- Resolving $\gamma^{\prime}$ precipitates down to $150 \mathrm{~nm}$, the spatial resolution of the SI images is as good as that of EDS performed at $10 \mathrm{kV}$.

- However, the total time required per pixel (acquisition and processing) for an iCHORD-SI map is approximately three times smaller than that required for an EDS- 
EBSD map at $10 \mathrm{kV}$ with 3,000 counts per spectrum. In addition, the iCHORD-SI acquisition time is much decreased compared to the EDS-EBSD method, and most the required time is spent in the processing of the experimental intensity profiles which is performed offline.

- The iCHORD-SI combination is a quite destructive method but the ion beam abrasion can be somewhat limited by reducing the number of acquired SE images and/or by adapting the scanning speeds, which may not degrade the quality of the results.

In this way, the iCHORD-SI combination is an interesting option for the $\gamma / \gamma^{\prime}$ microstructures which have a multimodal distribution of $\gamma^{\prime}$ precipitates from few micrometers to some tens of nanometers. Beyond superalloys, the iCHORD-SI combination may be used for any crystallographic system, where the phase discrimination is difficult/impossible by EBSD and where there is a sufficient contrast in atomic number between the phases.

\section{Acknowledgments}

The authors gratefully acknowledge the Orsay Physics Company (Fuveau, France) for the access to a FIB-SEM microscope and for their help to perform the iCHORD technique with it. This work received the financial support of the ANR-Safran industrial chair OPALE.

\section{Data availability}

The raw/processed data required to reproduce these findings cannot be shared at this time due to technical or time limitations.

\section{References}

[1] C. Langlois, T. Douillard, H. Yuan, N.P. Blanchard, A. Descamps-Mandine, B. Van de Moortèle, C. Rigotti, T. Epicier, Crystal orientation mapping via ion channeling: An alternative to EBSD, Ultramicroscopy. 157 (2015) 65-72. doi:10.1016/j.ultramic.2015.05.023.

[2] R.C. Reed, The Superalloys, Fundamentals and Applications, Cambridge University Press, 2006.

[3] A. Devaux, A. Helstroffer, J. Cormier, F. Pettinari-Sturmel, Effect of Aging Heat-Treatment on Mechanical Properties of AD730 ${ }^{\mathrm{TM}}$ Superalloy, 8th Int. Symp. Superalloy 718 Deriv. (2014). doi:10.1002/9781119016854.ch41.

[4] R.W. Cahn, P. Haseen, Physical Metallurgy, Volume I, North Holl, 1996.

[5] A. Porter, B. Ralph, The recrystallization of nickel-base superalloys, J. Mater. Sci. 16 (1981) 707-713. doi:10.1007/BF02402788.

[6] E. Nes, N. Ryum, O. Hunderi, On the Zener drag, Acta Metall. 33 (1985) 11-22. doi:10.1016/0001-6160(85)90214-7.

[7] F. Humphreys, M. Hatherly, Recrystallization and Related Annealing Phenomena (Second Edition), Elsevier, 2004. 
[8] K. Huang, K. Marthinsen, Q. Zhao, R.E. Logé, The double-edge effect of second-phase particles on the recrystallization behaviour and associated mechanical properties of metallic materials, Prog. Mater. Sci. 92 (2017) 284-359. doi:10.1016/j.pmatsci.2017.10.004.

[9] B. El-Dasher, A. Deal, Application of Electron Backscatter Diffraction to Phase Identification, in: A.J. Schwartz, M. Kumar, B.L. Adams, D.P. Field (Eds.), Electron Backscatter Diffr. Mater. Sci., Springer US, Boston, MA, 2009: pp. 81-95. doi:10.1007/978-0-387-88136-2_6.

[10] R.A. Schwarzer, J. Hjelen, Backscaterred Electron Imaging with an EBSD detector, Micros. Today. 23 (2015) 12-17. doi:10.1017/12 S1551929514001333.

[11] M.M. Nowell, S.I. Wright, Phase differentiation via combined EBSD and XEDS, J. Microsc. 213 (2004) 296-305. doi:10.1111/j.0022-2720.2004.01299.x.

[12] M.A. Charpagne, P. Vennéguès, T. Billot, J.M. Franchet, N. Bozzolo, Evidence of multimicrometric coherent $\gamma^{\prime}$ precipitates in a hot-forged $\gamma-\gamma^{\prime}$ nickel-based superalloy, J. Microsc. 263 (2016) 106-112. doi:10.1111/jmi.12380.

[13] C.A. Anderson, M.F. Hashler, Extension of electron microprobe techniques to biocemistry by the use of long wavelenght X-rays, in: R. Castaing, P. Deschamps, J. Philibert (Eds.), Proc. Fourth Int. Conf. X-Ray Opt. Microanal., Paris, 1966: pp. 310-327.

[14] I. Barkshire, P. Karduck, W.P. Rehbach, S. Richter, High-Spatial-Resolution Low-Energy Electron Beam X-Ray Microanalysis, Microchim. Acta. 132 (2000) 113-128. doi:10.1007/s006040050052.

[15] M.W. Phaneuf, Applications of focused ion beam microscopy to materials science specimens, Micron. 30 (1999) 277-288. doi:10.1016/S0968-4328(99)00012-8.

[16] A. Devaux, B. Picqué, M.F. Gervais, E. Georges, T. Poulain, P. Héritier, AD730TM - A new Nickel-based superalloy for high temperature engine rotative parts, Superalloys 2012. (2012) 911-919.

[17] L.C. Feldman, J.W. Mayer, S.T. Picraux, Materials Analysis by Ion Channeling, Submicron Crystallography, Academic Press, 1982.

[18] Y. Yahiro, K. Kaneko, T. Fujita, W.-J. Moon, Z. Horita, Crystallographic orientation contrast associated with $\mathrm{Ga}+$ ion channelling for $\mathrm{Fe}$ and $\mathrm{Cu}$ in focused ion beam method, J. Electron Microsc. (Tokyo). 53 (2004) 571-576. doi:10.1093/jmicro/dfh075.

[19] J. Schindelin, I. Arganda-Carreras, E. Frise, Fiji: an open-source platform for biological-image analysis, Nat. Methods. 9 (2012) 676-682. doi:10.1038/nmeth.2019.

[20] F. Bachmann, R. Hielscher, H. Schaeben, Grain detection from 2d and 3d EBSD data Specification of the MTEX algorithm, Ultramicroscopy. 111 (2011) 1720-1733. doi:10.1016/J.ULTRAMIC.2011.08.002. 
[21] D.C. Joy, Beam interactions, contrast and resolution in the SEM, J. Microsc. 136 (1984) 241258. doi:10.1111/j.1365-2818.1984.tb00532.x.

[22] T. Ishitani, H. Tsuboi, Objective Comparison of Scanning Ion and Scanning Electron Microscope Images, Scanning. 19 (1997) 489-497. doi:10.1002/sca.4950190707.

[23] D. Cohen-Tanugi, N. Yao, Superior imaging resolution in scanning helium-ion microscopy: A look at beam-sample interactions, J. Appl. Phys. 104 (2008). doi:10.1063/1.2976299.

[24] TESCAN, Secondary Ion TESCAN Detector, (n.d.). https://www.tescan.com/enus/technology/detectors/sitd.

[25] R. Gauvin, A Universal Equation for the Emission Range of X Rays from Bulk Specimens, Microsc. Microanal. 13 (2007) 354-357. doi:10.1017/S143192760707081X.

[26] M. Nowell, R. Anderhalt, T. Nylese, F. Eggert, R. de Kloe, M. Schleifer, S. Wright, Improved EDS Performance at EBSD Geometry, Microsc. Microanal. 17 (2011) 398-399. doi:10.1017/S1431927611002868.

[27] M. Charpagne, T. Billot, J. Franchet, N. Bozzolo, Heteroepitaxial Recrystallization Observed in René $65^{\mathrm{TM}}$ and Udimet $720^{\mathrm{TM}}$ : a New Recrystallization Mechanism Possibly Occurring in All Low Lattice Mismatch $\gamma-\gamma^{\prime}$ Superalloys?, Superalloys 2016. (2016) 417-426. doi:10.1002/9781119075646.ch45. 